\title{
A Study to Evaluate Clinical and Bacteriological Profile and the Effect of the Treatment to Diabetic Foot Ulcer Patients Both in Outpatient and Hospitalized Settings in a Newly Set Up Diabetic Foot Care Unit
}

\author{
Tamoghna Maiti1 ${ }^{1}$, Ratul Banerjee 2 , Sonai Mandal ${ }^{3}$, Amrita Panda ${ }^{4}$, Somenath Das ${ }^{5}$
}

\author{
${ }^{1}$ Department of Pharmacology, Bankura Sammilani Medical College, Bankura, West Bengal, India. ${ }^{2}$ Department \\ of Pharmacology, Bankura Sammilani Medical College, Bankura, West Bengal, India. ${ }^{3}$ Department of \\ Pharmacology, Bankura Sammilani Medical College, Bankura, West Bengal, India. ${ }^{4}$ Department of \\ Pharmacology, Bankura Sammilani Medical College, Bankura, West Bengal, India. ${ }^{5}$ Department of \\ Pharmacology, Bankura Sammilani Medical College, Bankura, West Bengal, India.
}

\section{ABSTRACT}

\section{BACKGROUND}

The problem of diabetes continues to explode in our country today. India now has the dubious distinction of being called, "the diabetic capital of the world". Diabetic foot ulcerations \& infections are one of the leading causes of mortality \& morbidity from diabetes. It is the most expensive complication of diabetes \& the leading cause of hospitalization when compared to other complication of diabetes. The number of cases \& problems associated with diabetic foot infection have dramatically increased in the recent years. In rural Bengal, the problem is grave as detection and treatment initiation is very late.

\section{METHODS}

A prospective observational study was conducted in Medicine \& Surgery OPD and IPD of Bankura Sammilani Medical College for twenty weeks. Baseline characteristic were noted, ulcer classification was done followed by swab culture, debridement and antimicrobial therapy. Then outcome was observed.

\section{RESULTS}

Maximum patients were aged between 38 to 58 years, were male and were Wagner 1-3 classification. From culture report $S$. aureus was predominant followed by Enterobacteriaceae group and anaerobes. Amoxicillin-clavulanic acid combination was the most effective antibiotic followed by amikacin. Treatment was satisfactory with dressing, debridement and appropriate antibiotic.

\section{CONCLUSIONS}

S. aureus, Enterococcus, Pseudomonas aeruginosa, E. coli \& Anaerobes were the most common causes of diabetic foot infections in my study, and were sensitive to the conventional antibiotics indicating that there is no evidence to suggest significant resistance to these antibiotics. Hence, their empirical usage, either oral or injectable (depending on the type of foot ulcer) is justified. Proper education regarding footwear \& foot care is strongly recommended in such patients.

\section{KEY WORDS}

Foot Ulcer, Diabetes, Swab Culture, Wagner Classification
Corresponding Author: Dr. Ratul Banerjee, Department of Pharmacology, Bankura Sammilani Medical College, Bankura, West Bengal, India.

E-mail:dr.ratulbanerjee@gmail.com

DOI: $10.14260 /$ jemds/2020/34

Financial or Other Competing Interests: None.

How to Cite This Article:

Maiti T, Banerjee R, Mandal S, et al. A study to evaluate clinical and bacteriological profile and the effect of the treatment to diabetic foot ulcer patients both in outpatient and hospitalized settings in a newly set up diabetic foot care unit. J. Evolution Med. Dent. Sci. 2020;9(03):152156, DOI: $10.14260 / \mathrm{jemds} / 2020 / 34$

Submission 19-11-2019, Peer Review 30-12-2019, Acceptance 06-01-2020, Published 20-01-2020.

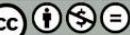




\section{BACKGROUND}

A diabetic foot infection is most simply defined as any inframalleolar infection in a person with diabetes mellitus.[1] Spectrum of infection includes paronychia, cellulitis, myositis, abscesses, necrotizing fasciitis, septic arthritis, tendonitis, and osteomyelitis. The wound may progress to become actively infected, and, by contiguous extension, the infection can involve deeper tissues. This sequence of events can be rapid (occurring over days or even hours), especially in an ischemic limb. Various poorly characterized immunologic disturbances, especially those that involve polymorph nuclear leukocytes, may affect some diabetic patients, and these likely increase the risk and severity of foot infections. ${ }^{[2,3,4]}$ Magnitude of diabetes mellitus is increasing globally at an alarming rate. About 150-170 million populations are suffering from this disease worldwide and the prevalence of diabetes will be double by 2025 as per WHO reports. Foot infections in diabetes are rarely due to a single organism. Aerobic bacteria (Staphylococcus spp., Streptococcus spp., and some species of Enterobacteriaceae), anaerobic flora (Bacteroides spp., Clostridium spp., and Peptostreptococci spp.), and some fungi are isolated most often.[5] India is called "The Diabetic Capital of the World". More than 30 million individuals are affected by diabetes in India. India is ahead of China and USA which are in $2^{\text {nd }}$ and $3^{\text {rd }}$ place respectively. Diabetic foot ulceration and infection are one of the leading causes of mortality and morbidity. It is the leading cause of hospitalization than any other complication of diabetes.

Diabetic foot ulcerations \& infections are one of the leading causes of mortality \& morbidity. It is the most expensive complication of diabetes \& the leading cause of hospitalization than any other complication of diabetes. The number of cases \& problems associated with diabetic foot infection has dramatically increased in the recent years. The main reason for this increase is growing diabetic population in the younger groups, disease is itself detected late, poor Glycaemic control, habits of bare foot walking, use of poor footwear, lack of awareness for foot care \& foot hygiene, significant delay in seeking treatment. Diabetic Foot Ulcer is caused mainly by neuropathy \& or Ischaemia, primarily from macro vascular disease \& frequently complicated by infection. These ulcers are usually in the areas of the foot which encounters repetitive trauma and pressure sensations. [6]

Diabetic neuropathy causes loss of protective sensation. Both of these contribute to loss of proprioception, poor balance, and lack of awareness of pain in the feet and lower limbs of patients with DM. Ulcers usually begin as small areas of pressure or irritation or from a minor trauma that is not perceived by the insensate neuropathic patient.[7,8] The pathogenesis of foot ulcer is complex, clinical presentation is variable \& its management requires early expert assessment. The aim of the therapy should be early intervention to allow prompt healing of the lesion \& prevent recurrence once it is healed. Intervention should be directed towards infection control, glycaemic control, peripheral ischaemia management \& abnormal pressure loading management caused by peripheral neuropathy. Diabetic foot ulcers are common and estimated to affect $15 \%$ of all diabetic individuals during their lifetime. It is now appreciated that $15-20 \%$ of patients with such foot ulcers go on to need an amputation. Almost $85 \%$ of the amputations are preceded by diabetic foot ulcers. ${ }^{[9,10,11]}$ The risk of lower leg amputations is $15-46$ times higher in the diabetics than in persons who do not have diabetes. Chronic osteomyelitis in patients with diabetes mellitus is the most difficult infection to cure. Adequate surgical debridement, in addition to antimicrobial therapy, is necessary to cure chronic osteomyelitis. Foot ulcer is the end stage complication of neuropathy \& or peripheral vascular disease. The risk of progression to this stage is far greater than the risk of progression to end stage sequel of retinopathy or nephropathy.

\section{METHODS}

After obtaining approval from the Institutional Ethics Committee, a prospective observational study was conducted from January 2018 to May 2018 at the outpatient as well as in-patient departments of Departments of Medicine and Surgery at Bankura Sammilani Medical College. Men and women with Type 2 diabetes mellitus within the age group of 30 to 60 yrs., studied for diabetic foot ulcer. We have included all patients we encountered with the said problem within the study period as our aim was to observe their parameters. A total of 15 patients with diabetic foot, both hospitalized as well as outpatients we found in the study who met our inclusion and exclusion criteria. Informed consent was taken from each patient. Also, the synopsis submitted to the ethical committee of Bankura Sammilani Medical College and we got the clearance to conduct the study. The foot ulcerations were graded using the Wagner classification. This classification suggested by Wagner accounts only for wound depth and appearance and widely used for classification of Diabetic foot ulcer.

\section{Inclusion Criteria}

- $\quad$ Adult male and female (age 40 yrs. -60 yrs.).

- Having foot ulcer in a known diabetic patient.

\section{Exclusion Criteria}

- Peripheral vascular disease without diabetes.

- Moribund patient.

- Patient not willing to sign in informed consent form.

- Below 40 yrs., and above 60 yrs.

Age, sex, type \& duration of diabetes, glycaemic control, presence of neuropathy, nephropathy (presence of microalbuminuria or macro-albuminuria), retinopathy, peripheral vascular disease (ischaemic symptoms \& intermittent claudication or rest pain, with or without absence of pedal pulses), duration \& size of ulcers, clinical outcome were noted on each patient. Clinical assessment for signs of infection (swelling, exudates, surrounding cellulitis, odour, tissue necrosis \& pyrexia) was made. Investigations were done according to the diabetic clinic protocol. They included complete blood count, fasting blood sugar, post-prandial blood sugar, random blood sugar, glycosylated haemoglobin, lipid profile, serum urea \& creatinine, liver function test, urine for micro-albumin/macro-albumin, X-ray of the wound, E.C.G. \& fundus examination. Ulcer size was determined by 
multiplying the longest \& widest diameters \& expressed in Centimetre's square. Osteomyelitis was diagnosed on suggestive changes in the radiographs. All the cases were monitored until discharge from the hospital.

Culture specimen were taken at the time of admission, after the surface of the wound had been washed vigorously by saline $\&$ followed by debridement of superficial exudates. Specimens were obtained by scrapping the ulcer base. The soft tissue specimens were promptly sent to the laboratory \& processed for aerobic \& anaerobic bacteria. Antimicrobial susceptibility testing of isolates was performed. All the patients/subjects were started empirical antibiotic (amoxycillin \& Metronidazole) therapy. Antibiotics were adopted based on the results of culture sensitivity report.

Statistical analysis was done with SPSS-23 and GraphPad Prism Ver. 6. Simple arithmetic process was done in the study.

\section{RESULTS}

The demographic characteristics of 15 patients with Diabetic Foot are shown in Table. L. The mean age of the subjects was 52 yrs. \& the mean duration of diabetes were 8 yrs. Nearly 7 patients $(46.67 \%)$ had diabetic foot lesion for more than 1 month before presentation at the hospital. In general, the patients were of old age \& had been on oral hypoglycaemic agents. The recommended glycaemic control was not seen in any of the 15 patients. All of them had Type 2 Diabetes. Males were predominant in the study group. All diabetic foots were classified \& grouped according to the Wagner Ulcer classification system. In the Wagner Classification system, foot lesions are divided into six grades $(0.5)$ based on the depth of wound \& extent of tissue necrosis. Wagner ulcer classification system shown in Table. 2. In my study all the patients were graded 1 to 3 in the Wagner classification. The details of the patients according to Wagner classification in our study is nine patients (60\%) were in Wagner grade-2, four patients $(26.67 \%)$ were in Wagner grade-3 \& two patients $(13.33 \%)$ were in Wagner grade-1.

Complication of diabetes in our study are as below-12 patients(80\%) had neuro pathy, 8 patients(53.33\%) had hypertension, 8 patients(53.33\%) had retinopathy, 6 patients (40\%) had nephropathy, 4 patients $(26.67 \%)$ had PVD \& 3 patients $(20 \%)$ had IHD, shown in Figure no. 1. The microbiological profile of the subjects is shown in Figure. 2. Staphylococcus aureus (40\%), Escherichia coli (13.33\%), Pseudomonas aeruginosa (13.33\%), Enterococcus (13.33\%) \& Anaerobes $(6.67 \%)$ were isolated in the study group. These microbiologically isolated patients were also evaluated for their antibiotic sensitivity pattern, which is shown in Figure. 3. Amoxicillin \& clavulanic acid combination was most sensitive antibiotic probably due to skin and soft tissue are full of gram-positive organism, followed by amikacin as our study second common bacteria was E. coli. In cephalosporin group ceftriaxone and cephalexin were effective. Cloxacillin and another aminoglycoside gentamycin covered the rest. We did not get any MRSA in our study. Ill-fitting shoe related factors, barefoot walking, neglected calluses were found to be the most common cause for the development \& worsening of foot lesions.

\begin{tabular}{|c|c|c|}
\hline Features & No. of Patients & Percentage \\
\hline Age (yrs.) & 3 & $20 \%$ \\
\hline$<40$ & 3 & $20 \%$ \\
\hline $40-50$ & 9 & $60 \%$ \\
\hline $50-60$ & No. of patients & Percentage \\
\hline Duration of Ulcer & 3 & $20 \%$ \\
\hline Less than 15 days & 5 & $33.33 \%$ \\
\hline Less than 30 days & 7 & $66.67 \%$ \\
\hline More than 30 days & 9 & $40 \%$ \\
\hline Male & 6 & \\
\hline Female & 52 yrs. & \\
\hline Average Age & $38-58$ yrs. & \\
\hline Range of Age & Table 1. Demographic Characteristics of the Patients (N=15) \\
\hline \multicolumn{2}{|c}{}
\end{tabular}

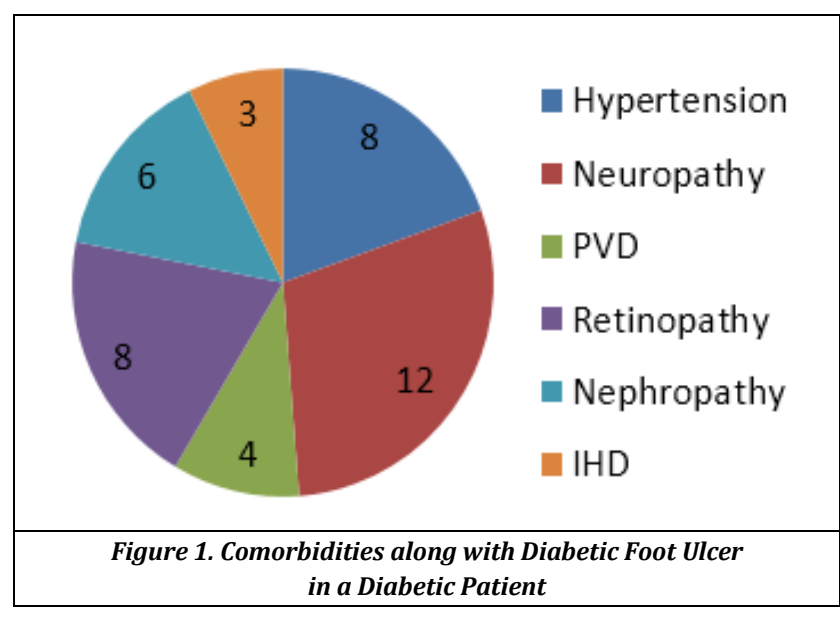

\begin{tabular}{|c|c|}
\hline Ulcer Grade & Definition \\
\hline 0 & $\begin{array}{r}\text { At risk foot-no ulceration-has bony } \\
\text { prominences/callus/deformities, etc. }\end{array}$ \\
\hline 1 & Superficial full thickness ulceration-not infected \\
\hline 2 & Deep ulceration-exposing tendons-no bone involvement \\
\hline 3 & $\begin{array}{c}\text { Deep ulceration with osteomyelitis-check with a probe, correlate } \\
\text { with X-ray findings }\end{array}$ \\
\hline 4 & Local gangrene-toes or forefoot \\
\hline 5 & Gangrene of whole foot \\
\hline \multicolumn{2}{|c|}{ Table 2. Wagner Ulcer Classification System } \\
\hline
\end{tabular}

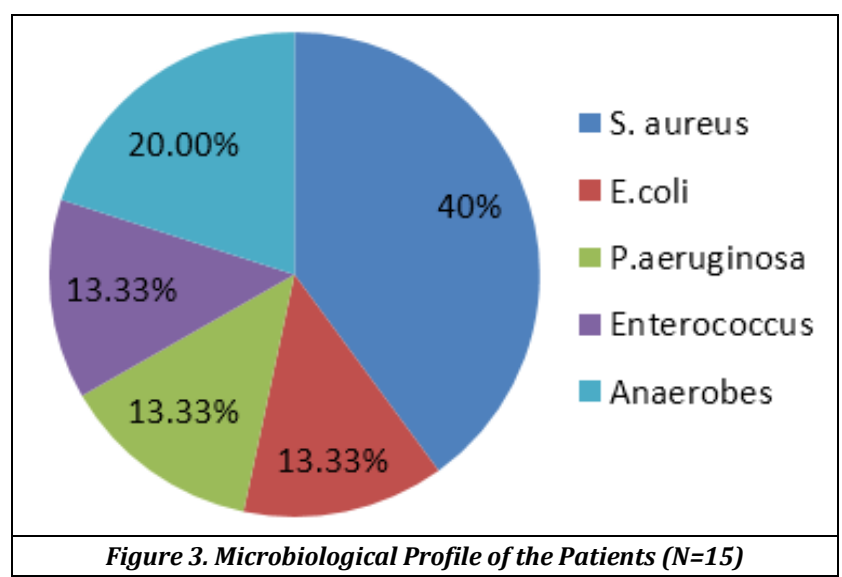

Figure 3. Microbiological Profile of the Patients ( $N=15)$ 


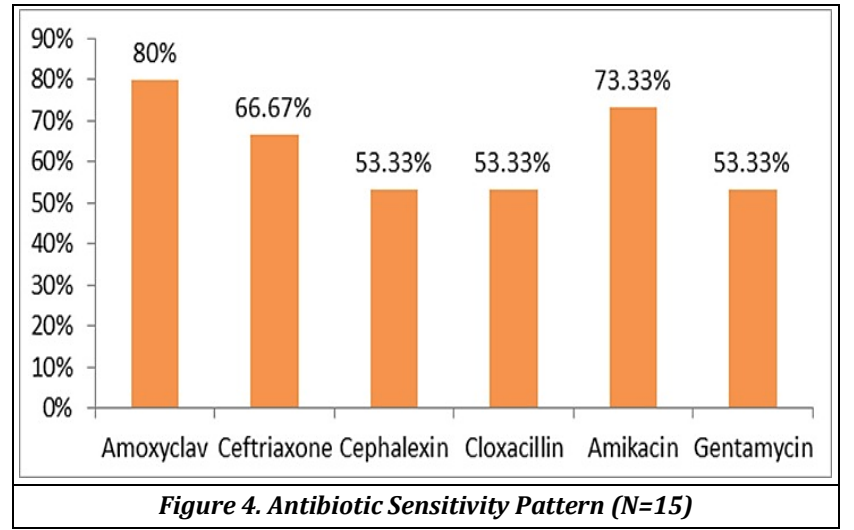

\section{DISCUSSION}

There are many studies regarding diabetic foot ulcer, but in rural Bengal where patient awareness and consciousness is concerned, where there is poverty is concerned their diabetic foot ulcer is very common. But they rarely seek physician's attention and also not aware of diabetes, detected late and also don't come for follow-up. So, we have gathered those few patients' data who came for cure of a severe complication of diabetes. Breen JD et al[12] shows, the common organisms seen in a diabetic foot ulcer are Staphylococcus aureus, Streptococcus, Pseudomonas aeruginosa, and rarely E. coli. Diabetes patients have higher carriage rate of Staphylococcus aureus in the nares and skin, and this increases the chances of infection of the ulcer. In our study we have got almost same result plus few anaerobe infections. Al Ayed MY et al[13] have found that the most common gram-negative pathogen identified was Pseudomonas aeruginosa (15.6\%), followed by Klebsiella spp. (6.7\%). The most common gram-positive pathogen was Staphylococcus aureus (35\%), followed by Streptococcus (8.9\%). The most widely accepted antibiotic to which the gram-negative bacteria were sensitive was gentamycin $(20.1 \%)$, followed by ciprofloxacin $(19 \%)$. The most routinely used antibiotic in the treatment of the grampositive bacteria was erythromycin (16\%), with trimethoprim-sulfamethoxazole (14.1\%) being the next most effective. In conclusion, Pseudomonas aeruginosa, Klebsiella spp., Staphylococcus aureus, and Streptococcus were the most common causes of diabetic foot infections. In our study gentamycin was not so useful, may be due to geographical location of the said study. The highest number of culturepositive cases was observed in Wagner grade 2 DFUs (45\%). Overall infection was monomicrobial in $83.20 \%$ (104) and polymicrobial in $16.80 \%$ (21) of samples. Staphylococcus aureus (21.09\%) and Pseudomonas aeruginosa (19.05\%) were the most common isolates. Linezolid (100\%) and imipenem $(75.70 \%)$ were the most effective antimicrobial agents against gram-positive and gram-negative isolates, respectively in a study done by Jaju $\mathrm{K}$ et al,[14] also differ from our result may be due to the same reason. We did not get any MRSA in our study which was fortunate to us and the patients, but it is a little bit controversial from other studies. The reason behind it may be the rural population consume very less antibiotic because of lifestyle and lack of availability which stood beneficial to them.

\section{CONCLUSIONS}

S. aureus, Enterococcus, Pseudomonas aeruginosa, E. coli \& Anaerobes were the most common causes of diabetic foot infections in this study, and were sensitive to the conventional antibiotics indicating that there is no evidence to suggest significant resistance to these antibiotics. Hence their empirical usage, either oral or injectable (depending on the type of foot ulcer) is justified. Proper education regarding footwear \& footcare is strongly recommended in such patients. It is recommended to treat diabetes as early as possible by means early treatment initiation.

\section{ACKNOWLEDGEMENT}

Authors thank Dr Shyamal Kundu, HOD. Department of Medicine, and Dr S. M. Naser, HOD. Department of Pharmacology and also to Associate Professor Dr. Ananya Mandal and Assistant Professor Dr. Tanmoy Gangopadhyay for their co-operation in present work and also grateful to all non-medical staffs of Medicine OPD and Pharmacology Department of Bankura Sammilani Medical College for their help in present work. Authors are also thankful to all PGTs and other faculty of Pharmacology Department of the same institution.

\section{REFERENCES}

[1] Armstrong DG, Lipsky BA. Advances in the treatment of diabetic foot infections. Diabetes Technol Ther 2004;6(2):167-77.

[2] Schubert S, Heesemann J. Infections in diabetes mellitus [In German]. Immun Infekt 1995;23(6):200-4.

[3] Gin H. Infection and diabetes [in French]. Rev Med Interne 1993;14(1):32-8.

[4] Joshi N, Caputo GM, Weitekamp MR, et al. Infections in patients with diabetes mellitus. $\mathrm{N}$ Engl J Med 1999;341(25):1906-12.

[5] Sharma VK, Khadka PB, Joshi A, et al. Common pathogens isolated in diabetic foot infection. Kathmandu Univ Med J 2006;4(3):295-301.

[6] Singer AJ, Tassiopoulos A, Kirsner RS. Evaluation and management of lower-extremity ulcers. N Engl J Med 2018;378(3):302-3.

[7] Vinkel J, Lohse N, Hyldegaard 0 . The clinical use of hyperbaric oxygen in the treatment of Danish patients with diabetic foot ulcers. Dan Med J 2019;66(2):A5528.

[8] Ennis WJ, Huang ET, Gordon H. Impact of hyperbaric oxygen on more advanced Wagner grades 3 and 4 diabetic foot ulcers: matching therapy to specific wound conditions. Adv Wound Care (New Rochelle) 2018;7(12):397-407.

[9] Palumbo PJ, Melton LJ. Peripheral vascular disease and diabetes. In: Harris MI, Hamman RF, eds. Diabetes in America. Washington: US Government Printing Office 1985: p. 16-21. NIH Pub. No. 85-1468.

[10] Pendsey S. Diabetic foot: a clinical atlas. New Delhi: Jaypee Brothers Medical Publishers 2003. 
[11] Pecoraro RE, Reiber GE, Burgess EM. Pathways to diabetic limb amputation: basis for prevention. Diabetes Care 1990;13(5):513-21.

[12] Breen JD, Karchmer AW. Staphylococcus aureus infections in diabetic patients. Infect Dis Clin North Am 1995;9(1):11-24.
[13] Al Ayed MY, Ababneh M, Robert AA, et al. Common pathogens and antibiotic sensitivity profiles of infected diabetic foot ulcers in Saudi Arabia. Int J Low Extrem Wounds 2018;24:1534734618793557.

[14] Jaju K, Pichare A, Davane M, et al. Profile and antibiotic susceptibility of bacterial pathogens associated with diabetic foot ulcers from a rural area. Wounds 2019;31(6):158-62. 